\title{
OSTRÁCODOS (CRUSTACEA) DE LA FORMACIÓN ALLEN (CRETÁCICO TARDÍO), CUENCA NEUQUINA, ARGENTINA
}

\author{
ANA PAULA CARIGNANO \\ División Paleozoología Invertebrados, Facultad de Ciencias Naturales y Museo. Universidad Nacional de La Plata, \\ Paseo del Bosque s/n, 1900 La Plata, Buenos Aires, Argentina. apcarignano@fcnym.unlp.edu.ar \\ JULIO ADRIAN VARELA \\ Facultad de Ciencias Exactas y Naturales, Universidad Nacional de La Pampa, Av. Uruguay 151, 6300 Santa Rosa, \\ La Pampa, Argentina. julioadrianvarela@hotmail.com
}

\begin{abstract}
OSTRACODES (CRUSTACEA) FROM THE ALLEN FORMATION (LATE CRETACEOUS), NEUQUÉN BASIN, ARGENTINA. A new microfossil association was found in the Allen Formation at Loma Puntuda (Neuquén Basin, Río Negro Province, Argentina). It is represented by scarce charophyta and numerous carapaces and valves of ostracods from the families Ilyocyprididae (such as Ilyocypris riograndensis and several species of Neuquenocypris) and Limnocytheridae (with the species Wolburgiopsis neocretacea and Vecticypris sp.). Molluscs, fragments of dinosaur eggshells and a plesiosaur remains had been registered previously in the same profile. This kind of association of non marine with a few marine fauna seems to indicate that the Allen Formation at Loma Puntuda was deposited in a shallow, fresh to oligohaline water body, near the coast.
\end{abstract}

Key words: late Campanian-early Maastrichtian, Limnocytheridae, Ilyocyprididae, Neuquén Basin.

\begin{abstract}
RESUMO - Dá-se a conhecer uma nova associação de microfósseis para a Formação Allen na localidade de Loma Puntuda (bacia de Neuquén, província de Rio Negro, Argentina). A mesma encontra-se representada por escassas carófitas e numerosas valvas e carapaças de ostracodes pertencentes às famílias Ilyocyprididae (como Ilyocypris riograndensis e várias espécies de Neuquenocypris) e Limnocytheridae (com as espécies Wolburgiopsis neocretacea e Vecticypris sp.). No mesmo perfil foram anteriormente registrados moluscos, fragmentos de cascas de ovo de dinossauros e restos de plesiossauro. Esta mistura de fauna continental com alguns elementos marinhos indica que a Formação Allen, em Loma Puntuda, foi depositada num corpo de água doce a oligohalino, pouco profundo, muito próximo à costa.
\end{abstract}

Palavras-chave: Neocampaniano-Eomaastrichtiano, Limnocytheridae, Ilyocyprididae, bacia de Neuquén.

\section{INTRODUCCIÓN}

El conocimiento micropaleontológico de la Formación Allen se debe principalmente a los trabajos de Musacchio (1973), Angelozzi (1980), Ballent (1980), Kielbowicz (1980), Musacchio \& Simeoni (1989, 1991), Ballent \& Carignano (2008) y Carignano \& Ballent (2009), quienes describen una variada microfauna, principalmente compuesta por ostrácodos de ambientes dulceacuícolas y salobres. En las cercanías del área de estudio, Rossi de García \& Malumián (in Caminos, 2001) mencionan asimismo un conjunto de ostrácodos lacustres. Esta área, ubicada en la región central de la provincia de Río Negro, se caracteriza por la presencia de una serie de "bajos" alineados en sentido noroeste-sudeste que frecuentemente preservan salinas o salitrales y muestran afloramientos de la Formación Allen.

En este trabajo se da a conocer una nueva asociación microfaunística para esta unidad, proveniente de la localidad Loma Puntuda (Figura 1), en las cercanías del área del Salitral de Santa Rosa (sector oriental de la Cuenca Neuquina). Se describen e ilustran las especies reconocidas y se realizan inferencias paleoecológicas para interpretar el ambiente de depositación de los sedimentos portadores de esta asociación. Esto último permite mejorar el conocimiento sobre la paleogeografía del norte de Patagonia durante las etapas iniciales de la primera ingresión procedente del océano Atlántico.

La microfauna recuperada está representada por caparazones y valvas de ostrácodos de buena preservación (Tabla 1). Conforman una asociación moderadamente diversa, de ambiente continental, constituida por seis especies pertenecientes a las familias Ilyocyprididae y Limnocytheridae. Acompañando a estos ostrácodos se han recuperado algunos dientes y vértebras de peces y escasos girogonites de carofitas muy aplastados y regularmente preservados.

\section{CONTEXTO GEOLÓGICO}

La Cuenca Neuquina (Figura 2A) se ubica en el sector centro-oeste de la Argentina, entre $\operatorname{los} 32^{\circ}$ y $41^{\circ}$ de latitud sur, extendiéndose por las actuales provincias de Neuquén, 


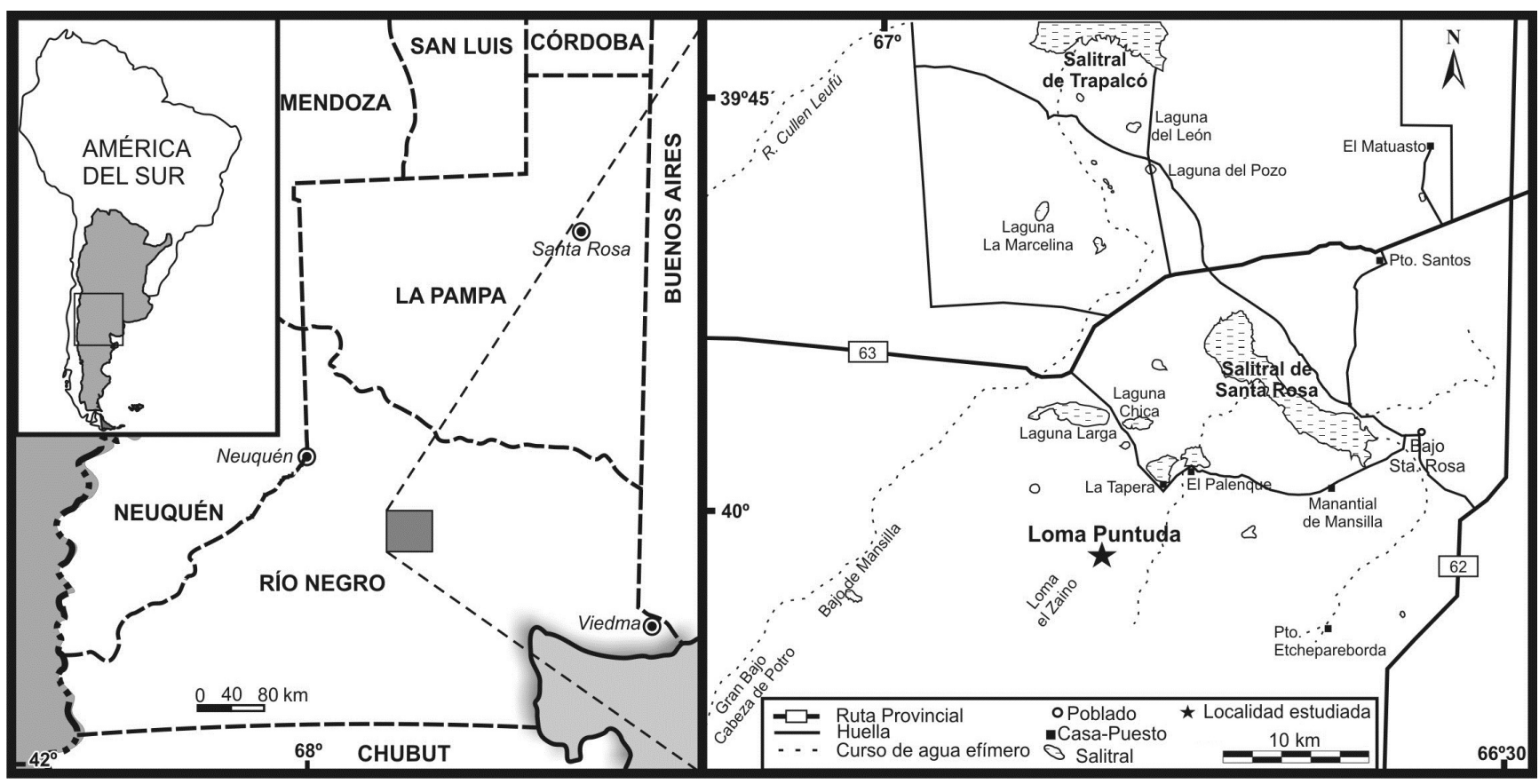

Figura 1. Mapa de ubicación de la localidad de estudio (modificado de Salgado et al., 2007).

Figure 1. Location map of the studied locality (modified from Salgado et al., 2007).

Tabla 1. Número de ejemplares por muestra.

Table 1. Number of specimens by sample.

\begin{tabular}{lccccccc}
\hline & M2 & M4 & M5 & M6 & M7 & M9 & M10 \\
\hline N. tenuipunctata & 13 & $3 ?$ & 4 & 54 & 498 & - & - \\
N. nahuelniyuensis & - & - & - & 5 & 6 & 152 & 10 \\
N. calfucurensis & - & - & - & - & 32 & - & - \\
I. riograndensis & 2 & - & - & - & 182 & - & - \\
W. neocretacea & - & 45 & 14 & 147 & 169 & - & 3 \\
Vecticypris sp. & - & - & - & - & 10 & - & - \\
\hline
\end{tabular}

Río Negro, La Pampa y Mendoza. Dentro de esta cuenca se reconocen dos regiones principales: la región Andina en el oeste y el engolfamiento Neuquino hacia el este y el sureste (Howell et al., 2005). Algunos autores proponen que el extremo norte de la cuenca llega hasta los $31^{\circ} \mathrm{S}$ (provincia de San Juan), llamando sector Aconcagua al área que va desde esa latitud hasta los $34^{\circ} \mathrm{S}$ y denominando a la cuenca como Neuquina-Aconcagüina (Aguirre-Urreta, 2001). Los márgenes noreste y sur de la cuenca limitan con las amplias áreas cratónicas del Macizo de Sierra Pintada y el Macizo Nordpatagónico respectivamente, mientras que el límite oeste está representado por el arco volcánico Andino (Howell et al., 2005). El basamento de la cuenca está constituido por rocas metamórficas, plutónicas, volcánicas y sedimentarias cuyas edades oscilan entre el Precámbrico y el Triásico Tardío (Digregorio \& Uliana, 1980; Franzese et al., 2007; Tunik et al., 2010). El relleno sedimentario de la misma se formó durante el
Triásico Tardío-Paleoceno Temprano e incluye sedimentitas silicoclásticas marinas y continentales, carbonatos y evaporitas que se acumularon durante distintas fases de evolución tectónica (e.g. Digregorio \& Uliana, 1980; Uliana \& Dellapé, 1981; Legarreta \& Gulisano, 1989; Legarreta \& Uliana, 1999). La última de estas fases comienza en el Cretácico Tardío cuando evoluciona hacia una cuenca de antepaís (foreland), depositándose las sedimentitas de los grupos Neuquén y Malargüe (Tunik et al., 2010).

La Formación Allen, unidad basal del Grupo Malargüe, suprayace a los depósitos continentales del Grupo Neuquén y es cubierta por los depósitos marinos de la Formación Jagüel (Figura 2B). Aflora en las provincias de Neuquén, Río Negro y La Pampa y su edad se asigna al Campaniano tardío-Maastrichtiano temprano en base a estudios magnetoestratigráficos y micropaleontológicos realizados en las unidades infra- y suprayacentes (Náñez \& Concheyro, 1997; Dingus et al., 2000). En la provincia de Mendoza, sedimentitas equivalentes son conocidas con el nombre de Formación Loncoche.

La localidad de Loma Puntuda se encuentra situada a los $40^{\circ} 1$ ' $44^{\prime \prime S}$ y $66^{\circ} 49^{\prime} 24,4$ "O, al sudoeste del Salitral de Santa Rosa, en la Provincia de Río Negro. En esta localidad, la Formación Allen está representada por una sucesión estratigráfica de $15 \mathrm{~m}$ de espesor, de base y techo cubiertos (Figura 3). Comienza con areniscas finas masivas, las cuales se encuentran bioturbadas y son portadoras de cáscaras de huevos de dinosaurios. Continúa una sucesión de pelitas masivas o finamente laminadas con algunos sectores con laminación heterolítica, entre las que se intercalan dos estratos de areniscas con estratificación entrecruzada. El primero, de areniscas medias, contiene bivalvos y gasterópodos asignados a Diplodon sp. y Paleoanculosa sp., restos de 


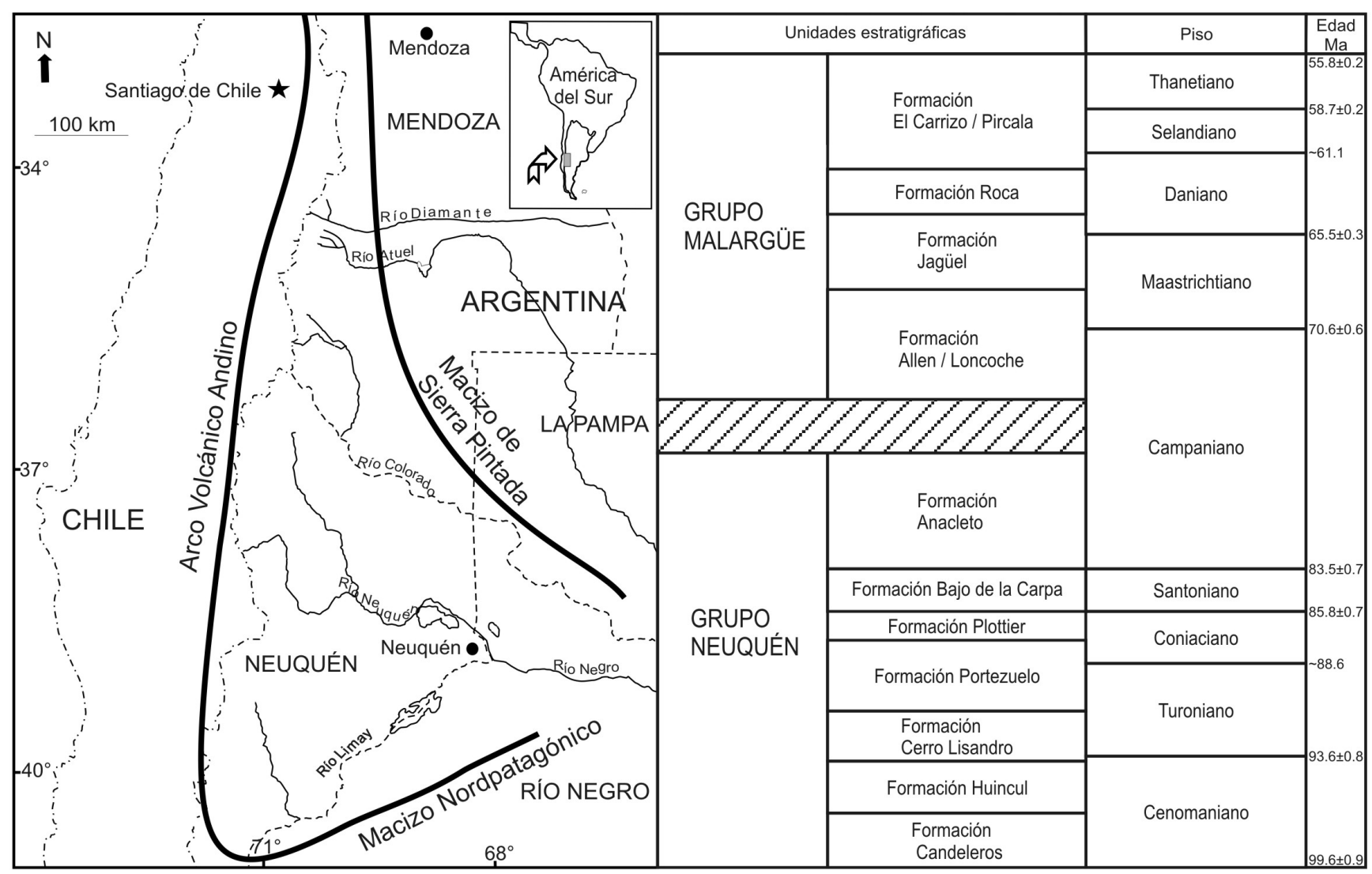

Figura 2. A, Ubicación y límites de la Cuenca Neuquina (modificado de Howel et al., 2005); B, Cuadro estratigráfico del Cretácico SuperiorPaleoceno de la Cuenca Neuquina (modificado de Leanza \& Hugo, 2001; edades en Ma de Ogg et al., 2008).

Figure 2. A, Location and limits of the Neuquén Basin (modified from Howel et al., 2005); B, Stratigraphic chart of the Upper CretaceousPaleocene from Neuquén basin (modified from Leanza \& Hugo, 2001; ages in My from Ogg et al., 2008).

vertebrados y cáscaras de huevos de dinosaurios. El segundo, de areniscas finas, suprayace a una capa pelítica de techo notablemente bioturbado y es portador de restos de un plesiosaurio perteneciente a la familia Polycotylidae (Salgado et al., 2007). La sección culmina con un estrato de $30 \mathrm{~cm}$ de yeso.

\section{MATERIAL Y MÉTODOS}

Fueron procesadas ocho muestras de la localidad Loma Puntuda, de las cuales siete resultaron fértiles, utilizando agua oxigenada al 10\%, luego lavadas a través de tamiz de malla 63 $\mu \mathrm{m}$ y por último secadas en estufa a $30^{\circ} \mathrm{C}$. Se realizó la extracción (picking) de ejemplares en aproximadamente $2 \mathrm{~g}$ de muestra seca, de toda la fracción retenida (> $63 \mu \mathrm{m})$. En las descripciones sistemáticas para ostrácodos a nivel supragenérico se ha adoptado la clasificación propuesta en Martin \& Davis (2001). A nivel genérico se siguieron los trabajos de Bertels (1972), Musacchio (1973, 1989) y Musacchio \& Simeoni (1989, 1991).

Las medidas y abreviaturas utilizadas son: L, largo; A, alto; a, ancho. Las dimensiones se indican en milímetros ( $\mathrm{mm}$ ) usándose la siguiente convención de tamaños: muy pequeño $(<0,400)$, pequeño $(0,401-0,500)$, mediano $(0,501-0,700)$; grande $(0,701-0,900)$, muy grande $(>0,901)$. El material queda depositado en la Universidad Nacional del Comahue, en la colección de Paleontología-Microfósiles, bajo la sigla UNC-PMIC.

\section{SISTEMÁTICA PALEONTOLÓGICA}

Orden PODOCOPIDA Sars, 1866

Suborden CYPRIDOCOPINA Jones, 1901

Superfamilia CYPRIDOIDEA Baird, 1845

Familia ILYOCYPRIDIDAE Kaufmann, 1900

Género Ilyocypris Brady \& Norman, 1889

Ilyocypris riograndensis Musacchio \& Simeoni, 1991

(Figuras 4A-C)

1991 Ilyocypris riograndensis Musacchio \& Simeoni, p. 357358, figs. 5, figs. 7.4,7.7,7.9.

Material y repositorio. 141 valvas y 43 caparazones, UNCPMIC 62 a 68.

Descripción. Caparazón de tamaño mediano a pequeño, inequivalvo, con la valva izquierda mayor que la valva derecha. Contorno en vista lateral subrectangular, con el borde dorsal subrectilíneo e inclinado hacia atrás, borde ventral suavemente cóncavo en la parte media posterior, borde anterior ampliamente redondeado y borde posterior redondeado, más bajo que el anterior. En vista dorsal de contorno elíptico, angostándose anteriormente y con el ancho mayor en la mitad posterior, coincidente en ambas valvas con la posición del tubérculo posterior. Ángulos cardinales redondeados pero distinguibles, mayor altura coincidente con el ángulo cardinal anterior. Ornamentación 
constituida por dos surcos subverticales bien marcados, de los cuales el posterior alcanza la zona media del caparazón. Estos surcos delimitan hacia adelante, entre ellos y por detrás tres elevaciones que se desarrollan en tubérculos, bien visibles en vista dorsal. Los tubérculos anterior y posterior son los mejor desarrollados, el tubérculo posterior además inclina un poco hacia el borde posteroventral. Superficie con un retículo poligonal

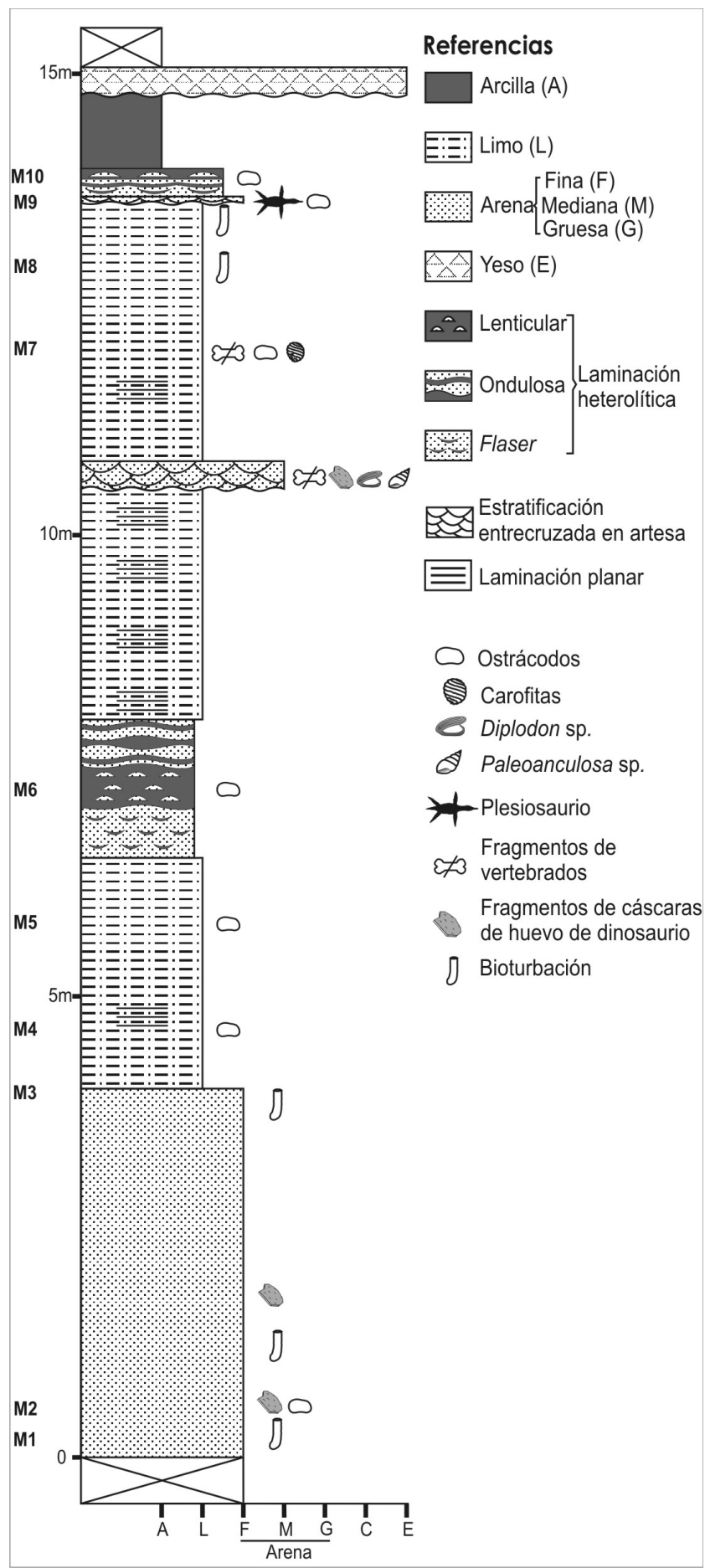

Figura 3. Columna estratigráfica de la Formación Allen en la localidad Loma Puntuda (modificada de Salgado et al., 2007).

Figure 3. Stratigraphic column of the Allen Formation at Loma Puntuda (modified from Salgado et al., 2007). paralelo a los márgenes de las valvas. Caracteres internos: lamela interna calcificada moderadamente ancha en las áreas anterior y posteroventral, lists bien visibles. Charnela adonta.

Dimensiones de los ejemplares ilustrados. Figura 4. A, UNC-PMIC 62, L= 0,545 mm, A= 0,304 mm; B, UNC-PMIC 63, L= 0,529 mm, a = 0,201 mm; C, UNC-PMIC 64, L=0,525 $\mathrm{mm}, \mathrm{A}=0,293 \mathrm{~mm}$.

Distribución. Ilyocypris riograndensis fue definida originalmente para sedimentitas no marinas en El Zampal (provincia de Mendoza, Argentina), en las formaciones Plottier (Coniaciano tardío), Bajo de la Carpa (Santoniano) y Anacleto (Campaniano). Formas semejantes han sido citadas también en el Cretácico Superior de Brasil, en las formaciones Araçatuba (Campaniano-Maastrichtiano temprano) por Gobbo-Rodrigues et al. (1999a) y Adamantina (TuronianoSantoniano) por Dias-Brito et al. (2001).

Observaciones. Se diferencia del material tipo descrito por Musacchio \& Simeoni (1991) del Cretácico Superior de Mendoza, Argentina en detalles de la ornamentación (tubérculo posterior más desarrollado, presencia de pequeñas papilas rodeando los tubérculos) y en su menor tamaño. En comparación con las formas brasileñas, Musacchio (in DiasBrito et al., 2001) señala que los ejemplares argentinos, sobre todo los provenientes de la Formación Anacleto, poseen el tubérculo posterior poco desarrollado, el reticulado muy delicado y el contorno de las valvas más trapezoidal, características que coinciden con las observadas en los ejemplares aquí estudiados. Las formas brasileñas, al parecer, se asemejan más a las registradas para las formaciones Plottier y Bajo de la Carpa en El Zampal (Mendoza, Argentina).

Género Neuquenocypris Musacchio, 1973

Neuquenocypris calfucurensis Musacchio, 1973 (Figuras 4D-F)

1973 Ilyocypris (Neuquenocypris) calfucurensis Musacchio, p. 17-20, lám. V, figs. 1-2, 10, 14, lám. VI, figs. 1-7,9.

1989 Neuquenocyopris calfucurensis n. comb. Musacchio, p. 835 .

Material y repositorio. 32 valvas y algunos fragmentos. UNCPMIC 69 a 71.

Descripción. Caparazón grande, frágil, inequivalvo, con la valva izquierda menor que la valva derecha. Contorno en vista lateral subtrapezoidal redondeado, borde dorsal recto inclinado hacia atrás, borde anterior ampliamente redondeado, más alto que el posterior, borde ventral levemente cóncavo y borde posterior redondeado. Ángulos cardinales bien marcados, mayor altura coincidente con el ángulo cardinal anterior. En vista dorsal de contorno subelíptico, borde anterior acuminado, borde posterior truncado, mayor ancho en la parte media. Depresión anterodorsal en la que se observan dos surcos suaves. El caparazón está fuertemente ornamentado, la ornamentación primaria está constituida por una depresión anterodorsal en la que se observan dos suaves surcos subverticales y pústulas irregularmente distribuidas, que pueden dispersarse más allá de la zona de los surcos. Flanqueando los surcos dos tubérculos bajos, el tubérculo posterior es de mayor tamaño y se encuentra 
un poco por debajo del anterior. La superficie de las valvas está cubierta por hoyuelos que conforman una especie de reticulado paralelo a los márgenes. Presencia de espinas truncadas en la punta, dispuestas regularmente siguiendo el contorno de las valvas e irregularmente dispuestas hacia el centro de las mismas. Estas espinas varían en forma, desde mamelares hasta cónicas y pueden o no tener el ápice crateriforme. Denticulaciones marginales en los bordes anterior, ventral y posterior y espinas ensanchadas en la punta acompañan el contorno de las valvas, mejor desarrolladas en la valva izquierda. Caracteres internos: charnela adonta, lamela interna calcificada bien desarrollada anteriormente, con vestíbulo. Demás caracteres no observados. Dimensiones de los ejemplares ilustrados. Figura 4. D, UNCPMIC 69, L=0,848 mm, A=0,506 mm; E, UNC-PMIC 70, L=0,830 $\mathrm{mm}, \mathrm{A}=0,494 \mathrm{~mm} ; \mathrm{F}, \mathrm{UNC}-\mathrm{PMIC} 71, \mathrm{~L}=0,882 \mathrm{~mm}, \mathrm{~A}=0,507 \mathrm{~mm}$. Distribución. Especie descripta originalmente para el Campaniano tardío (Formación Anacleto) en Paso Córdoba y cerca de Vista Alegre, provincias de Río Negro y Neuquén respectivamente, Argentina, asociada a ostrácodos no marinos y carofitas (Musacchio, 1973; Musacchio \& Simeoni, 1989, 1991). Observaciones. Neuquenocypris calfucurensis es bien distinguible de otras especies del mismo género por su compleja ornamentación. Los ejemplares de Loma Puntuda son un poco más pequeños que los de la localidad tipo.

\section{Neuquenocypris tenuipunctata Musacchio \& Simeoni, 1991 \\ (Figuras 4G-L, W)}

1991 Neuquenocypris tenuipunctata Musacchio \& Simeoni, p. 370-371, figs. $10.1,10.2,10.5-10.7$.

Material y repositorio. 512 valvas y 55 caparazones, UNCPMIC 72 a 85.

Descripción. Caparazón de tamaño grande, arriñonado, bien calcificado y moderadamente hinchado, inequivalvo con la valva derecha un poco más grande que la valva izquierda. Contorno en vista dorsal subelíptico, con el ancho mayor en la mitad posterior, bordes anterior y posterior acuminados. En vista lateral de contorno subrectangular redondeado, borde anterior ampliamente redondeado, borde dorsal recto, suavemente inclinado hacia atrás hasta casi horizontal, borde posterior redondeado, borde ventral levemente cóncavo en la parte media, más notoriamente en la valva izquierda. Ángulos cardinales bien marcados, mayor altura coincidente con el ángulo cardinal anterior. Depresión anterodorsal con dos surcos poco definidos, subverticales, algo inclinados hacia atrás, que no llegan al centro de las valvas. Superficie de las valvas cubierta por un suave puncteado sin orientación definida, con una serie de denticulaciones marginales en los bordes anterior, ventral y posterior, más desarrolladas en la valva izquierda y pequeñas papilas, mejor desarrolladas en el margen anterior, dispersas e irregularmente distribuidas. Juveniles de contorno subtrapezoidal, con el borde dorsal marcadamente inclinado hacia atrás. Caracteres internos: lamela interna calcificada bien desarrollada, con numerosos canales marginales rectos y vestíbulo amplio. Impresiones musculares con la distribución típica del género. Charnela formada en la valva derecha por una barra que se ensancha en los extremos.
Dimensiones de los ejemplares ilustrados. Figura 4. G, UNC-PMIC 72, L= 0,771 mm, A=0,435 mm; H-I, UNC-PMIC 73, L=0,868 mm, A= 0, 530 mm; J, UNC-PMIC 74, L=0,428 $\mathrm{mm}, \mathrm{A}=0,270 \mathrm{~mm}$; K, UNC-PMIC 75, L=0,698 mm, a=0,307 $\mathrm{mm}$; L, UNC-PMIC 76, L=0,759 mm, A=0,440 mm; W, UNCPMIC 77, L=0,817 mm, A=0,449 mm.

Distribución. La presente especie fue hallada originalmente en sedimentitas continentales del Campaniano tardíoMaastrichtiano temprano (Formación Allen) en la localidad Nahuel Niyeu, provincia de Río Negro, Argentina (Musacchio \& Simeoni, 1991).

Observaciones. Musacchio \& Simeoni (1991) en la descripción original mencionan que el mayor desarrollo de las denticulaciones marginales se observa en la valva derecha; en el material estudiado en este trabajo, esta relación es inversa. Una forma similar es Neuquenocypris nahuelniyuensis Musacchio, 1989, también presente en los niveles estudiados, la cual se diferencia por la presencia de papilas cubriendo la superficie del caparazón, una depresión anterodorsal bien delimitada, con surcos subverticales más profundos y ángulos cardinales menos definidos y en el contorno en vista dorsal, en donde se observa el borde posterior truncado. Por otro lado, $N$. tenuipunctata es muy semejante al género Cyprideamorphella Mandelstam, 1956 (in Moore \& Pitrat, 1961) del Cretácico Superior de Asia, ambos poseen caparazón con forma arriñonada, charnela con una barra que se ensancha en los extremos, cubrimiento inverso y superficie de las valvas puncteada. Sin embargo, no se observan en el material estudiado las costillas submarginales presentes en Cyprideamorphella.

\section{Neuquenocypris nahuelniyuensis Musacchio, 1989} (Figuras 4M-N)

1989 Neuquenocypris nahuelniyuensis Musacchio, p. 386, lám. 4, figs. 7-12.

Material y repositorio. 118 caparazones y 55 valvas. Material ilustrado UNC-PMIC 86 a 90.

Descripción. Caparazón mediano, de contorno subrectangular, algo elongado, inequivalvo con la valva derecha mayor que la valva izquierda. En vista dorsal caparazón de contorno subelíptico, con el borde anterior angosto y el posterior casi truncado, ancho mayor coincidente con la mitad posterior. En vista lateral, borde dorsal recto, un poco inclinado hacia atrás, borde anterior redondeado, borde ventral ligeramente cóncavo, borde posterior redondeado, ángulo cardinal anterior bien marcado, coincide con la mayor altura. Dos surcos anterodorsales bien definidos, inclinados hacia la región posterior, el surco posterior es más largo y llega hasta la zona de las impresiones musculares. Ornamentación de las valvas constituida por un puncteado neto y papilas regularmente distribuidas, sobre todo en las zonas anterior y posterior. En el área de la depresión anterodorsal, el puncteado posee hoyuelos más profundos y no se observan papilas. Denticulaciones marginales en las áreas anterior, ventral y posterior. Caracteres internos: lamela interna calcificada moderadamente ancha, charnela como en $N$. tenuipunctata.

Dimensiones de los ejemplares ilustrados. Figura 4. M, UNC-PMIC 86, L= 0,690 mm, a= 0,304 mm; N, UNC-PMIC $87, \mathrm{~L}=0,653 \mathrm{~mm}, \mathrm{~A}=0,353 \mathrm{~mm}$. 
Distribución. Especie reconocida en el Campaniano tardíoMaastrichtiano temprano (Formación Allen), localidades Nahuel Niyeu y Treneta, en la provincia de Río Negro, Argentina (Musacchio \& Simeoni, 1991).

Observaciones. Los especímenes de Loma Puntuda parecen tener una depresión anterodorsal y surcos más definidos y profundos que aquellos hallados en Nahuel Niyeu y Treneta. Neuquenocypris nahuelniyuensis es muy semejante a Neuquenocypris zampalensis (Angelozzi) sobre todo en la zona de los surcos anterodorsales, que se exhiben bien definidos y algo inclinados hacia atrás, sin embargo esta especie posee tres tubérculos poco prominentes que no son observados en el material estudiado.

Suborden CYTHEROCOPINA Baird, 1850

Superfamilia CYTHEROIDEA Baird, 1850

Familia LIMNOCYTHERIDAE Klie, 1938

Subfamilia LIMNOCYTHERINAE Klie, 1938

Género Wolburgiopsis Musacchio, 1978

Wolburgiopsis neocretacea (Bertels, 1972)

(Figuras 4O-Q)

1972 Wolburgia? neocretaea Bertels, p. 181-182, lám. 1, figs. 8a-b, fig. 2.

1975 "Wolburgia" neocretacica Bertels. Musacchio y Palamarczuk, p. 308, lám. I, fig. 20.

1978 Wolburgiopsis neocretacea $\mathrm{n}$. comb. Musacchio. Uliana \& Musacchio, p. 126, lám. II, figs. 41-44.

Material y repositorio. 297 caparazones, 81 valvas, UNC-PMIC 91 a 98.

Descripción. Caparazón de tamaño pequeño, de valvas subiguales, la valva izquierda un poco mayor que la derecha, sobrepasando a esta en los bordes anterior y posterior. Dorsalmente se observa que la valva derecha cubre a la izquierda a lo largo de la zona charnelar. De contorno subrectangular en vista lateral, con el borde anterior redondeado, borde posterior truncado y algo expandido a redondeado en la mitad inferior, borde dorsal recto, borde ventral cóncavo en la mitad posterior. En vista dorsal de contorno subelíptico, acuminado anteriormente y más ancho en la mitad posterior. Ángulos cardinales bien marcados, mayor altura coincidente con el ángulo cardinal anterior. Ornamentación constituida por dos surcos anterodorsales oblicuos, algo inclinados hacia el borde anterior, suavemente delimitados y superficie de las valvas con un reticulado grueso, sin ordenamiento preferencial. Ventralmente además, se observa una débil cresta. Poros normales escasos. Caracteres internos: charnela constituida por dos dientes lobulados y un surco medio en la valva derecha. Valva izquierda con dos fosetas lobuladas y una barra crenulada? que se ensancha un poco en los extremos. Lamela interna calcificada moderadamente ancha. Demás caracteres no observados.

Dimensiones de los ejemplares ilustrados. Figura 4. O, UNC-PMIC 91, L= 0, $335 \mathrm{~mm}, \mathrm{~A}=0,165 \mathrm{~mm}$; P, UNC-PMIC 92, $\mathrm{L}=0,365 \mathrm{~mm}, \mathrm{a}=0,125 \mathrm{~mm}$; Q, UNC-PMIC 93, $\mathrm{L}=0,339$ $\mathrm{mm}, \mathrm{a}=0,128 \mathrm{~mm}$.

Distribución. En Argentina esta especie fue reconocida en sedimentitas no marinas del Cretácico Tardío de las formaciones Plottier, Bajo de la Carpa y Anacleto en El Zampal, provincia de Mendoza y Loncoche, en la localidad de Huantraico, provincia de Neuquén (Bertels, 1972; Uliana \& Musacchio, 1978; Musacchio \& Vallati, 2007). En Brasil, en el Cretácico Tardío de la Cuenca de Paraná, formaciones Araçatuba y Adamantina (Gobbo-Rodrigues et al., 1999b; Dias-Brito et al., 2001).

Observaciones. Los ejemplares aquí estudiados poseen surcos más someros que los descriptos por Bertels (1972) en el material tipo, además los caparazones son de menor tamaño. Por otro lado, si bien no se menciona en la descripción original, en este caso se pudo observar dimorfismo de probable origen sexual en las valvas, reconociéndose algunas más largas y angostas (machos?) y otras más anchas y cortas (hembras?) (Figuras 4P-Q).

\section{Subfamilia TIMIRIASEVIINAE Mandelstam, 1960 Género Vecticypris Keen, 1972}

Vecticypris sp. (Figuras 4R-V)

Material y repositorio. 10 valvas y fragmentos, UNC-PMIC 99 a 101.

Descripción. Caparazón mediano, frágil, de contorno subromboidal redondeado, muy hinchado, con forma de corazón en vista posterior, subtriangular en vista dorsal con el borde posterior truncado y el borde anterior acuminado. En vista lateral borde dorsal muy convexo, borde anterior redondeado, algo truncado, borde ventral suavemente convexo, borde posterior redondeado. Ancho mayor por detrás de la mitad de las valvas, mayor altura en la mitad del caparazón, márgenes de las valvas incurvados ventralmente, excepto en el margen anterior. Superficie de las valvas cubierta con un puncteado neto que se hace paralelo al margen ventral de las valvas, dándole el aspecto de finas estrías. Caracteres internos: lamela interna calcificada angosta en la zona anterior, más delgada posteriormente, que posee en la valva izquierda selvage bien desarrollado. En el margen de la valva derecha, se distingue un reborde. Charnela constituida en la valva derecha por un alveolo anterior alargado, una barra media lisa, aproximadamente del mismo largo que el alveolo anterior, alveolo posterior no observado. Valva izquierda con un diente posterior, que nace del selvage, diente anterior no observado.

Dimensiones de los ejemplares ilustrados. Figura 4. R-T, UNC-PMIC 99, $\mathrm{L}=0,626 \mathrm{~mm}, \mathrm{~A}=0,414 \mathrm{~mm}, \mathrm{a}=0,231 \mathrm{~mm}$; $\mathrm{U}$, UNC-PMIC 100, L= 0,556 mm, a= 0,247 mm; V, UNC-PMIC $101, A=0,384 \mathrm{~mm}, \mathrm{a}=0,432 \mathrm{~mm}$.

Distribución. Campaniano tardío-Maastrichtiano temprano (Formación Allen), localidad Loma Puntuda, Río Negro, Argentina.

Observaciones. Colin et al. (2000) reúnen las especies primitivas de "Metacypris" con borde dorsal convexo dentro del género Vecticypris Keen, del Paleógeno del noroeste europeo. Vecticypris se caracteriza además por poseer una charnela con elementos terminales lisos y elongados y un elemento medio corto, un vestíbulo anterior débil y duplicadura con fuertes selvage y reborde (Keen, 1972; Colin et al., 2000). La presente especie guarda semejanzas con Vecticypris cf. polita (Grekoff) 
(in Dias-Brito et al., 2001) de la Formación Adamantina, en la forma general del caparazón, aunque los especímenes aquí estudiados son más triangulares en vista dorsal. Se diferencia de Vecticypris xestoleberiformis (Colin), del Cenomaniano de Francia en la ornamentación de las valvas y en el contorno en vista dorsal, que es más redondeado en la especie europea. Con Metacypris sp. C Schäfer, 2005 de depósitos de agua dulce del Mioceno inferior de Suiza, se asemeja sobretodo en el contorno en vista dorsal y en la ornamentación, aunque la especie aquí estudiada es menos inflada.

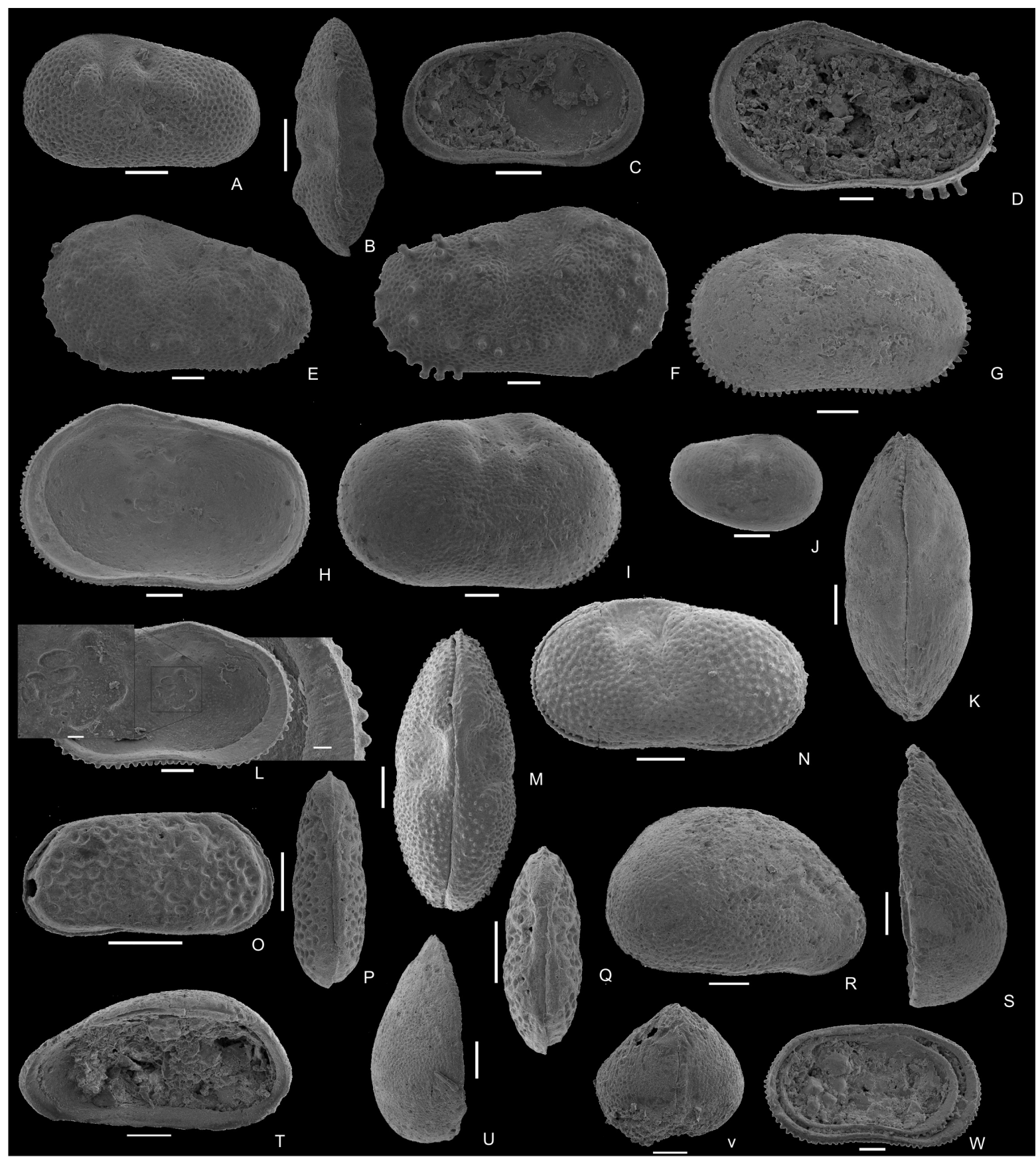

Figura 4. Ostrácodos de la Formación Allen, localidad Loma Puntuda. A-C, Ilyocypris riograndensis: A, UNC-PMIC 62, valva izquierda, vista externa; B, UNC-PMIC 63, caparazón vista dorsal; C, UNC-PMIC 64, valva izquierda, vista interna. D-F, Neuquenocypris calfucurensis: D, UNC-PMIC 69, valva derecha, vista interna; E, UNC-PMIC 70, valva izquierda, vista externa; F, UNC-PMIC 71, valva derecha, vista externa. G-L, W, Neuquenocypris tenuipunctata: G, UNC-PMIC 72, valva izquierda, vista externa; H-I, UNC-PMIC 73, valva derecha, H, vista interna, I, vista externa; J, UNC-PMIC 74, juvenil, valva derecha; K, UNC-PMIC 75, caparazón, vista dorsal; L, UNC-PMIC 76, valva izquierda, vista interna, detalle de impresiones musculares y lamela interna calcificada; W, UNC-PMIC 77, valvas imbricadas. M-N, Neuquenocypris nahuelniyuensis: M, UNC-PMIC 86 caparazón, vista dorsal; N, UNC-PMIC 87, caparazón vista lateral izquierda. O-Q, Wolburgiopsis neocretacea: O, UNC-PMIC 91, caparazón vista lateral derecha; P, UNC-PMIC 92, caparazón masculino vista dorsal; Q, UNC-PMIC 93, caparazón femenino, vista dorsal. R-V, Vecticypris sp.: R-T, UNC-PMIC 99, valva derecha, R, vista externa, S, vista dorsal, T, vista interna inclinada; U, UNC-PMIC 100, caparazón vista posterior; V, UNC-PMIC 101, valva izquierda, vista dorsal. Escalas =0,100 mm.

Figure 4. Ostracods from the Allen Formation, Loma Puntuda locality. Scale bars $=0.100 \mathrm{~mm}$. 


\section{DISCUSIÓN}

En Loma Puntuda, los ilyocyprididos están representados por Ilyocypris, género cosmopolita y muy común en ambientes dulceacuícolas y oligohalinos, y Neuquenocypris, género instituido por Musacchio para agrupar ilyocyprididos con, entre otras características, cubrimiento inverso y caparazón muy ornamentado. Este último ha sido reconocido en la Argentina desde el Aptiano hasta el Paleoceno, en sedimentos de origen no marino, muchas veces asociado a carofitas y otros ostrácodos de agua dulce (Musacchio \& Simeoni, 1991). El mayor número de especies de Neuquenocypris se registra en Argentina hacia el Cretácico más superior, coincidente con un decaimiento en la diversidad de Cypridea Bosquet. Neuquenocypris se encontró también en el Cretácico Tardío de Brasil (Dias-Brito et al., 2001) y Bolivia (Camoin et al., 1991). Asimismo se lo menciona en el Cretácico Tardío de Francia (Babinot et al., 1996) y Eslovaquia (Pipík et al., 2009).

Wolburgiopsis es un limnocytherido que contiene aproximadamente siete especies del Cretácico Temprano alto y Tardío, de ambientes continentales salobres y dulceacuícolas, casi siempre asociado con ostrácodos ilyocyprididos. Siguiendo a Colin \& Danielopol (1978) y Danielopol et al. (1989), se lo ubica dentro de la Subfamilia Limnocytherinae, Tribu Limnocytherini por las siguientes características: caparazón fuertemente calcificado, con dimorfismo sexual, hembras sin bolsa incubadora, surco vertical y charnela antimerodonta. Coincidiendo con Musacchio (1990), Wolbugiopsis parece conformar, junto a Looneyellopsis Krömmelbein y Huillicythere Musacchio un grupo definido de pequeños limnocytheridos con caparazón de contorno subtrapezoidal y charnela de tipo merodonta. $\mathrm{Su}$ distribución se restringe a Argentina (Bertels, 1972; Uliana \& Musacchio, 1978; Musachio \& Vallati, 2007), Brasil (GobboRodrigues et al., 1999b; Dias-Brito et al., 2001) y Bolivia (Rodrigo Gainza et al., 1990). Neuquenocypris nahuelniyuensis y Neuquenocypris tenuipunctata han sido halladas asociadas en sedimentos continentales de la Formación Allen en la zona de Nahuel Niyeu (Formación Coli Toro según Musacchio, 1989; Musacchio \& Simeoni, 1989, 1991) y Loma Puntuda, dos localidades de la provincia de Río Negro relativamente cercanas entre sí. Por otro lado, Neuquenocypris calfucurensis se registró en sedimentos continentales de la Formación Anacleto en las provincias de Río Negro y Neuquén, junto a carofitas y a otros cypridoideos no marinos (Musacchio, 1973; Musacchio \& Simeoni, 1991).

Ilyocypris riograndensis y formas similares del Cretácico Tardío parecen haber tenido una amplia distribución en Sudamérica en sedimentos de origen continental, ya que se las registra en varias formaciones tanto argentinas (formaciones Plottier, Bajo de la Carpa, Anacleto y Allen) como brasileñas (formaciones Araçatuba y Adamantina), asociadas a una rica variedad de ostrácodos y carofitas (Musacchio \& Simeoni, 1989, 1991; Gobbo-Rodrigues et al., 1999a; Dias-Brito et al., 2001; Musacchio \& Vallati, 2007).

La especie Wolburgiopsis neocretacea ha sido hallada junto a Ilyocypris argentiniensis Musacchio \& Simeoni, $1991 \mathrm{y}$ al foraminífero Discorbis sp. en el Cretácico Tardío (provincia de Mendoza, Argentina) conformando una asociación de ambiente salobre, aunque Musacchio (in Uliana \& Musacchio, 1978) señala la posible aloctonía de los especímenes de W. neocretacea. Bertels (1972) describe en sedimentos continentales de la sección inferior de la Formación Loncoche (provincia de Neuquén) una asociación constituida por oogonios de carofitas y los ostrácodos W. neocretacea, Ilyocypris triebeli Bertels, 1972 y Candona? huantraicoensis Bertels, 1972, depositados en facies lacustres y deltaicas. En la Formación Plottier, en El Zampal (provincia de Mendoza), Musacchio \& Vallati (2007) refieren asimismo un conjunto de carofitas y ostrácodos, entre los que mencionan a W.cf. neocretacea y a Ilyocypris cf. riograndensis, provenientes de depósitos de aguas someras y oligotróficas. Por otro lado, esta especie también ha sido hallada en la Formación Adamantina, depositada en un lago que en periodos de extrema aridez era separado en cuerpos más pequeños (DiasBrito et al., 2001). Al igual que I. riograndensis, W. neocretacea parece haber tenido una amplia distribución, tanto geográfica como temporal, en ambientes no marinos y de mezcla.

Las especies de ostrácodos y carofitas comunes a Brasil y Argentina han sido documentadas en numerosos aportes (véase Musacchio et al., 1996, 2002; Musacchio, 2001; Gobbo Rodrigues et al., 1999a,b; Dias-Brito et al., 2001). Con respecto a los factores intervinientes en la dispersión de los ostrácodos mesozoicos, Whatley (1990) hace una distinción entre factores extrínsecos e intrínsecos. Entre los primeros, en el caso de Argentina se menciona el rompimiento del aislamiento geográfico ocurrido a partir del Aptiano, debido a movimientos tectónicos que expusieron grandes áreas a la denudación (Musacchio et al., 1996; Musacchio, 2000, 2001). Esta nueva disposición paleogeográfica permitió el intercambio de especies entre distintas regiones, situación que continuó hasta el Cretácico Tardío. Entre los factores intrínsecos, es decir aquellos que hacen a la propia biología de los ostrácodos, la presencia de huevos resistentes a la desecación y congelamiento y el desarrollo de hembras partenogenéticas parecen haber sido claves en la dispersión de los cyprididos. La dispersión entonces, se habría producido por agentes tanto biológicos (en el tracto digestivo de peces o entrampados en el barro pegado al cuerpo de vertebrados) como abióticos (viento).

La abundancia de valvas y caparazones de adultos y juveniles bien preservadas sugiere que los ostrácodos de Loma Puntuda habrían sufrido poco transporte. Además, la presencia de valvas imbricadas (o estructura de tipo "cup in cup", Figura 4V) sugiere que la depositación se produjo en o cerca de los bordes de cuerpos de agua muy someros sometidos a suave oleaje (Wakefield, 1995) y ha sido reportada ya para la Formación Allen en las cercanías del lago Pellegrini (Carignano \& Ballent, 2009).

Respecto al total de individuos, el porcentaje de las 2-3 especies más abundantes ha sido utilizado para caracterizar asociaciones de distintos ambientes (Whatley, 1983). Según este autor, en ambientes dulceacuícolas o de mezcla varía generalmente entre el $70 \%$ y $90 \%$, mientras que en ambientes marinos es menor al 50\%. En el caso de Loma Puntuda el porcentaje fue del 70,3\%, tomando como especies más abundantes a Neuquenocypris tenuipunctata y Wolburgiopsis neocretacea.

En relación a la presencia de un plesiosaurio Polycotylidae en la localidad Loma Puntuda, son varias las menciones en la literatura de este tipo de reptil en ambientes no marinos y de mezcla, ejemplo de esto son los provenientes de la Formación 
Horseshoe Canyon (Campaniano-Maastrichtiano, Alberta, Canadá) y Ziliujing (Jurásico Temprano?, Chongqing, China). En estos yacimientos se sugiere que los individuos juveniles y subadultos habrían habitado en ambientes marinos y no marinos, mientras que los adultos habrían estado limitados al primero de estos (Sato et al. 2003, 2005; Sato \& Wu, 2006 y referencias allí citadas). Con respecto a los restos del plesiosaurio Polycotylidae de Loma Puntuda, Salgado et al. (2007) señalan que este ejemplar estaría representado por un individuo inmaduro, posiblemente un subadulto, situación que se condice con los hallazgos mencionados anteriormente. Otro ejemplo para Argentina lo constituyen los restos de un Polycotylidae hallados en la Formación La Colonia (Campaniano-Maastrichtiano, provincia de Chubut, Argentina) junto a otros vertebrados terrestres y de agua dulce (Gasparini \& de la Fuente, 2000).

\section{CONCLUSIONES}

La asociación microfaunística hallada puede ser interpretada entonces como de agua dulce a oligohalina, depositada en un cuerpo de agua somero, de baja energía y cercano a la costa, el cual posiblemente haya formado parte de un estuario. Las evidencias que apoyan esta idea son la presencia de ostrácodos ligados a ambientes continentales y el poco transporte que parecen haber sufrido los mismos, sumado a la ausencia de otros microfósiles típicamente marinos o de mezcla (foraminíferos, ostrácodos marinos); la presencia de valvas imbricadas, estructura generada en o cerca del borde de cuerpos de agua muy somera; la fauna acompañante hallada en las capas macrofosilíferas del afloramiento, constituida por los moluscos Diplodon sp. y Paleoanculosa sp., los cuales se consideran de ambientes salobres y/o dulceacuícolas, restos de cáscaras de huevos de dinosaurios y un plesiosaurio subadulto. Por último, se confirma para otra localidad la presencia de taxones en común con microfaunas brasileñas, situación ya planteada por diversos autores y se amplía la distribución geográfica y temporal de los ostrácodos Neuquenocypris calfucurensis, Wolburgiopsis neocretacea e Ilyocypris riograndensis.

\section{AGRADECIMIENTOS}

Los autores del trabajo desean agradecer J. O'Gorman y M.L.C. Marcilese por la lectura crítica del manuscrito. AS. Ballent y A. Parras por sus valiosos aportes. Alos revisores M.C. Cabral y J.P. Colin por sus útiles sugerencias y comentarios que ayudaron a enriquecer el presente trabajo. Este trabajo se realizó en el marco de los proyectos de CONICET (PIP 0819), de la Universidad Nacional de La Plata (N589) y de la Agencia de Promoción Científica y Tecnológica (BID 1728-PICT 357).

\section{REFERENCIAS}

Aguirre-Urreta, M.B. 2001. Marine Upper Jurassic-Lower Cretaceous stratigraphy and biostratigraphy of the AconcaguaNeuquén Basin, Argentina and Chile. Journal of Iberian Geology, 27:71-90.

Angelozzi, G.N. 1980. Dos nuevas especies de Ilyocypris (Ostracoda) de ambiente salobre del Cretácico superior en la cuenca del Neuquén, República Argentina. Ameghiniana, 17(2):163-167.

Babinot, J.-F.; Colin, J.-P. \& Tambareau, Y. 1996. Late Cretaceous non-marine ostracods from Europe: biostratigraphy, palaeobiogeography and taxonomy. Cretaceous Research, 17:151167. doi: $10.1006 /$ cres. 1996.0012

Ballent, S.C. 1980. Ostrácodos de ambiente salobre de la Formación Allen (Cretácico superior) en la Provincia de Río Negro (República Argentina). Ameghiniana, 17(1):67-82.

Ballent, S.C. \& Carignano, A.P. 2008. Morphological abnormalities in Late Cretaceous and early Paleocene foraminifer tests (Northern Patagonia, Argentina). Marine Micropaleontology, 67:288-296. doi:10.1016/j.marmicro.2008.02.003

Bertels, A. 1972. Ostrácodos de agua dulce del miembro inferior de la Formación Huantrai-Co (Maastrichtiano inferior), provincia del Neuquén, República Argentina. Ameghiniana, 9:173-182.

Caminos, R. 2001. Hoja Geológica 4166-1, Valcheta. Provincia de Río Negro. Buenos Aires, Instituto de Geología y Recursos Minerales, Servicio Geológico Minero Argentino, 71 p. (Boletín 310).

Camoin, G.; Rouchy, J.-M.; Babinot, J.-F.; Deconinck, J.-F. \& Tronchetti, G. 1991. Dynamique sedimentaire et evolution paléogéographique d'un basin continental en position d'arrièrearc: le Maastrichtien de la Cordillère Orientale (Bolivie). Comptes Rendus de l'Academie des Sciences de Paris, 312(2):1335-1341.

Carignano, A.P. \& Ballent, S.C. 2009. Microfósiles (Foraminifera, Ostracoda) y su respuesta a las variaciones paleoambientales. Un ejemplo en la Formación Allen (Cretácico Superior), Cuenca Neuquina, Argentina. Ameghiniana, 46(2):307-320.

Colin, J.P.; Cabral, M.C.; Dépêche, F. \& Mette, W. 2000. Sinuocythere n. gen. (Ostracoda, Limnocytheridae, Timiriaseviinae), a new genus of limnic ostracode from southern Tethyan Middle and Upper Jurassic. Micropaleontology, 46(2):123-134. doi: 10.2113/46.2.123

Colin, J.P. \& Danielopol, D.L. 1978. New data on the systematic of the Limnocytheridae (Ostracoda, Crustacea). Geobios, 11(4):563567. doi:10.1016/S0016-6995(78)80084-9

Danielopol, D.L.; Martens, K. \& Casale, L.M. 1989. Revision of the genus Leucocythere Kaufmann, 1892 (Crustacea, Ostracoda, Limnocytheridae), with the description of a new species and two new tribes. Bulletin de l'Institut Royal des Sciences Naturelles de Belgique, Biologie, 59:63-94.

Dias-Brito, D.; Musacchio, E.A.; Castro, J.C.; Maranhão, M.S.A.S.; Suárez, J.M. \& Rodrigues, R. 2001. Grupo Bauru: uma unidade continental do Cretáceo no Brasil - concepções baseadas em dados micropaleontológicos, isotópicos e estratigráficos. Revue de Paléobiologie, 20(1):245-304.

Digregorio, J.H. \& Uliana, M.A. 1980. Cuenca Neuquina. In: SIMPOSIO DE GEOLOGÍA REGIONAL ARGENTINA, 2, 1976. Anales, Córdoba, Academia Nacional de Ciencias, p. 985-1032.

Dingus, I.O.; Clarke, J.; Chiappe, L.M. \& Coria, R.A. 2000. Stratigraphy and Magnetostratigraphic/Faunal constraints for the age of Sauropod embryo-bearing rocks in the Neuquén Group (Late Cretaceous, Neuquén Province, Argentina). American Museum Novitates, 3290:1-11.

Franzese, J.R.; Veiga, G.D.; Muravchik, M.; Ancheta, M.D. \& D’Elía, L. 2007. Estratigrafía de "sin-rift" (Triásico Superior-Jurásico Inferior) de la Cuenca Neuquina en la sierra de Chacaico, Neuquén, Argentina. Revista Geológica de Chile, 34(1):49-62. doi: 10.4067/ S0716-02082007000100003

Gasparini, Z. \& de la Fuente, M. 2000. Tortugas y plesiosaurios de la Formación La Colonia (Cretácico Superior) de Patagonia, Argentina. Revista Española de Paleontología, 15(1):23-35.

Gobbo-Rodrigues, S.R.; Petri, S. \& Bertini, R.J. 1999a. Ocorrências de ostrácodes na Formação Araçatuba do Grupo Bauru, Crétaceo superior da Bacia do Paraná, e possibilidades de correlação com depósitos isócronos argentinos - Parte I: Família Ilyocyprididae. Acta Geologica Leopoldensia, 23(49):3-13.

Gobbo-Rodrigues, S.R.; Petri, S. \& Bertini, R.J. 1999b. Ocorrências de ostrácodes na Formação Araçatuba do Grupo Bauru, Crétaceo superior da Bacia do Paraná, e possibilidades de correlação com 
depósitos isócronos argentinos - Parte II: Familia Limnocytheridae. Revista Universidade Guarulhos, Geociências, 4(6):5-11.

Howell, J. A.; Schwarz, E.; Spalletti, L. A. \& Veiga, G. D. 2005. The Neuquén Basin: an overview. In: The Neuquén Basin, Argentina: a case study in sequence stratigraphy and basin dynamics, London, Geological Society, p.1-14 (Special Publications 252).

Keen, M.C. 1972. The Sannoisan and some other Upper Paleogene Ostracoda from north-west Europe. Palaeontology, 15(2):267-325.

Kielbowicz, A. 1980. Estudio de la microfauna de sedimentitas del Cretácico superior de la zona de lago Pellegrini, Provincia de Río Negro. Facultad de Ciencias Exactas y Naturales, Universidad de Buenos Aires, Trabajo Final de Licenciatura, 68 p.

Leanza, H.A. \& Hugo, C.A. 2001. Cretaceous red beds from southern Neuquén Basin (Argentina): age, distribution and stratigraphic discontinuities: In: INTERNATIONAL SYMPOSIUM ON MESOZOIC TERRESTRIAL ECOSYSTEMS, 7, 1999. Publicación Especial, Buenos Aires, Asociación Paleontológica Argentina, p. 117-122.

Legarreta, L. \& Gulisano, C.A. 1989. Análisis estratigráfico secuencial de la Cuenca Neuquina (Triásico Superior-Terciario Inferior). In: Cuencas Sedimentarias Argentinas, Tucumán, Facultad de Ciencias Naturales, Universidad Nacional de Tucumán, p. 221-243 (Serie Correlación Geológica 6).

Legarreta, L. \& Uliana, M.A. 1999. El Jurásico y Cretácico de la Cordillera Principal y la Cuenca Neuquina. 1. Facies Sedimentarias. In: Geología Argentina, Buenos Aires, Instituto de Geología y Recursos Minerales, p. 399-432 (Anales 29).

Martin, J.W. \& Davis, G.E. 2001. An updated classification of the Recent Crustacea. Los Angeles, National History Museum of Los Angeles County, $124 \mathrm{p}$.

Moore, R.C. \& Pitrat, C.W. (eds.). 1961. Treatise on Invertebrate Paleontology. Part Q. Arthropoda 3. Crustacea, Ostracoda. New York, Geological Society of America and University of Kansas Press, 442 p.

Musacchio, E.A. 1973. Charophytas y ostrácodos no marinos del Grupo Neuquén (Cretácico superior) en algunos afloramientos de las provincias de Río Negro y Neuquén, República Argentina. Revista del Museo de La Plata (nueva serie), Paleontología, 48:1-37.

Musacchio, E.A. 1989. Biostratigraphy of the non-marine Cretaceous of Argentina based on calcareous microfossils. In: J. Weidmann (ed.) Cretaceous of the Western Tethys, E. Schweizerbart'sche Verlagsbuchhandlung, p. 811-850.

Musacchio, E.A. 1990. Non-marine Cretaceous ostracods from Argentina and their palaeobiogeographical relationships. In: R. Whatley \& C. Maybury (eds.) Ostracoda and Global Events, British Micropalaeontological Society Publication Series/Chapman and Hall, p. 557-569.

Musacchio, E.A. 2000. Biostratigraphy and biogeography of Cretaceous charophytes from South America. Cretaceous Research, 21:211220. doi:10.1006/cres. 1999.0197

Musacchio, E.A. 2001. Relaciones paleobiogeográficas de los ostrácodos no marinos del Jurásico y el Cretácico de Patagonia. Acta Geologica Leopoldensia, 24(53/53):293-310.

Musacchio, E.A.; Dias-Brito, D.; Maranhão, M.S.A.S. \& Suárez, J.M. 2002. Comparando algas carófitos (algas verdes) neocretácicos del Grupo Bauru (Brasil) y de Argentina. In: SIMPÓSIO SOBRE O CRETÁCICO DO BRASIL, 6/ SIMPOSIO SOBRE EL CRETÁCICO DE AMÉRICA DEL SUR, 2, 2002. Resumos expandidos, Rio Claro, p. 329-334.

Musacchio, E.A. \& Simeoni, M. 1989. Cretaceous non-marine cypridacean Ostracoda from central and northern Argentine Patagonia. Courier Forschungsinstitut Senckenberg, 113:77-88.

Musacchio, E.A. \& Simeoni, M. 1991. Taxonomy of some Cretaceous non-marine ostracods of paleobiogeographical interest. Neues Jahrbuch für Geologie und Paläontologie Abhandlungen, 180(3):349-389.
Musacchio, E.A. \& Vallati, P. 2007. Late Cretaceous non marine microfossils of the Plottier Formation (Cretaceous) at Zampal, Argentina. Cuadernos del Museo Geominero, 8:273-278.

Musacchio, E.A.; Vallati, P. \& Simeoni, M. 1996. Microfósiles no marinos del Cretácico en Patagonia: sus relaciones con asociaciones brasileñas. In: SIMPÓSIO SOBRE O CRETÁCEO DO BRASIL, 4, 1996. Boletim, Rio Claro, UNESP p. 189-193.

Náñez, C. \& Concheyro, A. 1997. Límite Cretácico-Paleógeno. Provincia del Neuquén, Dirección Nacional del Servicio Geológico, Geología y Recursos Minerales del Departamento Añelo, p. 129-149 (Anales 25).

Ogg, J.G.; Ogg, G. \& Gradstein, F.M. 2008. The Concise Geologic Time Scale. Cambridge University Press, 177 p.

Pipík, R.; Sýkora, M.; Colin, J.-P. \& Havrila, M. 2009. A new phytal limnic ostracod Rosacythere carpathica sp. nov. from the Upper Cretaceous of the Western Carpathians: implications for evolution of the Timiriaseviinae. Cretaceous Research, 30:465-476. doi:10.1016/j.cretres.2008.09.001

Rodrigo Gainza, L.A.; Branisa, L. \& Musacchio, E.A. 1990. Las cuencas de los Grupos Puca y Tacuru (Cretácico-Terciario inferior) de Bolivia, con dos capítulos sobre algunos microfósiles calcáreos del Cretácico de Bolivia. In: W. Volkheimer (ed.) Bioestratigrafía de los Sistemas Regionales del Jurásico y Cretácico de América del Sur, Comité Sudamericano del Jurásico y Cretácico/Editorial Inca, p. 569-623.

Salgado, L.; Parras, A. \& Gasparini, Z. 2007. Un plesiosaurio de cuello corto (Plesiosauroidea, Polycotylidae) del Cretácico Superior del norte de Patagonia. Ameghiniana, 44(2):349-358.

Sato, T.; Eberth, D.A.; Nicholls, E.L. \& Manabe, M. 2005. Plesiosaurian remains form non-marine to paralic sediments. In: P.J Curie \& E.B. Kapelhus (eds) Dinosaur Provincial Park. A spectacular ancient ecosystem revealed, Indiana University Press, p. 249-276.

Sato, T.; Li, C. \& Wu, X.-C. 2003. Restudy of Bishanopliosaurus youngi Dong 1980, a fresh-water plesiosaurian from the Jurassic of Chongqing. Vertebrata PalAsiatica, 41(1):17-33.

Sato, T. \& Wu, X.-C. 2006. Review of plesiosaurians (Reptilia: Sauropterygia) from the Upper Cretaceous Horseshoe Canyon Formation in Alberta, Canada. Paludicola, 5(4):150-169.

Schäfer, P. 2005. Beiträge zur Ostracoden- und Foraminiferen- Fauna der Unteren Süßwassermolasse in der Schweiz und in Savoyen (Frankreich). 2*). La Chaux (Kanton Waadt, Schweiz). Senckenbergiana lethaea, 85(1):95-117.

Tunik, M.; Folguera, A.; Naipauer, M.; Pimentel, N. \& Ramos, V.A. 2010. Early uplift and orogenic deformation in the Neuquén Basin: constraints on the Andean uplift from $\mathrm{U}-\mathrm{Pb}$ and $\mathrm{Hf}$ isotopic data of detrital zircons. Tectonophysics, 489(1-4):258-273. doi:10.1016/ j.tecto.2010.04.017

Uliana, M.A. \& Dellapé, D.A. 1981.Estratigrafía y evolución paleoambiental de la sucesión maastrichtiana-eoterciaria del engolfamiento neuquino. (Patagonia Septentrional). In: CONGRESO GEOLÓGICO ARGENTINO, 8, 1981. Actas, San Luis, AGA, p. 673-711.

Uliana, M.A. \& Musacchio, E.A. 1978. Microfósiles calcáreos no marinos del Cretácico superior en Zampal, provincia de Mendoza, Argentina. Ameghiniana, 15(1-2):111-135.

Wakefield, M.I. 1995. Ostracod biostratinomy at lagoonal shorelines: examples from the Great Estuarine Group, Middle Jurassic, Scotland. Proceedings of the Geologists' Association, 106:211218. doi:10.1016/S0016-7878(08)80024-7

Whatley, R. 1983. The application of Ostracoda to palaeoenvironmental analysis. In: R.F. Maddocks (ed.) Applications of Ostracoda, University of Houston, p. 51-77.

Whatley, R. 1990. The relationships between extrinsic and intrinsic events in the evolution of Mesozoic non-marine Ostracoda. In: E. Kauffman \& O. Walliser (eds.), Extinction Events in Earth History, Springer-Verlag, p. 253-263.

Received in November, 2010; accepted in April, 2011. 